\title{
Delayed cervical palsy following cervical spine fusion leads to an increase in hospital-related costs
}

\author{
Ross C. Puffer, MD, ${ }^{1}$ William E. Clifton, MD, ${ }^{2}$ Grant W. Mallory, MD, ${ }^{1}$ and Michelle J. Clarke, MD ${ }^{1}$ \\ 1Department of Neurosurgery, Mayo Clinic, Rochester, Minnesota; and ${ }^{2}$ Morsani College of Medicine, University of South Florida, \\ Tampa, Florida
}

\begin{abstract}
OBJECT Delayed cervical palsy (DCP) is a known complication following cervical spine surgery. While most DCPs eventually improve, they can result in significant temporary disability. Postoperative complications affect hospital length of stay (LOS) as well as overall hospital cost. The authors sought to determine the hospital cost of DCP after cervical spine fusion operations.
\end{abstract}

METHODS A retrospective review of patients undergoing cervical fusion for degenerative disease at the Mayo Clinic from 2008 to 2012 was performed. Patients who developed DCPs not attributable to intraoperative trauma were included. All nonoperative-related costs were compared with similar costs in a control group matched according to age, sex, and surgical approach. All costs and services were reflective of the standard costs for the current year. Raw cost data were presented using ratios due to institutional policy against publishing cost data.

RESULTS There were 27 patients (18 men, 9 women) who underwent fusion and developed a DCP over the study period. These patients were compared with 24 controls ( 15 men, 9 women) undergoing fusion in the same time period. There was no difference between patients and controls in mean age $(62.4 \pm 3.1$ years vs $63.8 \pm 2.5$ years, respectively; $p=$ $0.74)$, LOS ( $4.2 \pm 3.3$ days vs $3.8 \pm 4.5$ days, respectively; $p=0.43$ ), or operating room-related costs $(1.08 \pm 0.09$ vs 1.0 \pm 0.07 , respectively; $p=0.58$ ). There was a significant difference in nonoperative hospital-related costs between patients and controls $(1.67 \pm 0.15$ vs $1.0 \pm 0.09$, respectively; $p=0.04$ ). There was a significantly higher utilization of postoperative imaging (CT or MRI) in the DCP group (14/27, 52\%) when compared with the matched cohort $(4 / 24,17 \% ; p=0.018)$, and a significantly higher utilization of physiatry services (24/27 [89\%] vs $15 / 24$ [63\%], respectively; $p=0.046)$.

CONCLUSIONS While DCPs did not significantly prolong the length of hospitalization, they did increase hospital-related costs. This method could be further extrapolated to model costs of other complications as well.

http://thejns.org/doi/abs/10.3171/2014.9.SPINE14166

KEY WORDS delayed cervical palsy; fusion; length of stay; hospital costs; operating room costs; control; postoperative imaging; physiatry

$\mathrm{P}$ OSTOPERATIVE delayed cervical palsies (DCPs) are a well-known risk of cervical spine procedures. . $^{1,2,4,5}$ While a single, cohesive origin has yet to be identified, there are multiple factors believed to be associated, including postoperative nerve root tethering, thermal injury from high speed drill use, foraminal stenosis from kyphotic deformity correction, spinal cord ischemia, and instability. ${ }^{2-7,10-12}$ Although DCPs most commonly affect the C-5 myotome unilaterally, they can involve essentially any cervical myotome or multiple myotomes, and are believed to occur bilaterally in $5 \%-7 \%$ of cases. ${ }^{3,7}$ The reported incidence is variable in the literature, ranging from $0 \%$ to $30 \%$ of cases depending on the index pathology and primary procedure, although the average incidence via meta-analysis is $4.6 \% .^{8,9}$ Delayed cervical palsies typically manifest within the first 2 weeks of surgery, but have been reported to present as late as 2 months postoperatively. Fortunately, the majority of DCPs improve within 6 months, although as many as 19\% may have permanent residual deficits. ${ }^{8}$

Postoperative complications may increase the length of stay (LOS) and the overall hospital costs with varying degrees depending on severity of the complication and associated diagnostic testing and further treatment. As reimbursement models continue to change, understanding the effect of postoperative complications is becoming es-

ABBREVIATIONS DCP = delayed cervical palsy; LOS = length of stay; OR = operating room. SUBMITTED February 11, 2014. ACCEPTED September 25, 2014.

INCLUDE WHEN CITING Published online October 31, 2014; DOI: 10.3171/2014.9.SPINE14166.

DISCLOSURE The authors report no conflict of interest concerning the materials or methods used in this study or the findings specified in this paper. 
sential as hospitals strive to deliver better care at lower costs. Using matched cohort analysis, we reviewed LOS and hospital-related costs to better understand the impact of DCPs in the perioperative period.

\section{Methods \\ Study Population}

Institutional review board approval from the Mayo Clinic was obtained for this study. All cervical spine procedures performed by the Department of Neurosurgery from 2008 to 2012 were recorded. These cases were further stratified into fusion operations and decompressiononly operations. A prospectively maintained morbidity and mortality log was reviewed for patients who underwent cervical fusion and developed postoperative weakness during the study period. This morbidity and mortality $\log$ is populated by the surgical residents and staff surgeons who performed the cases, as well as the postoperative hospital management. Follow-up evaluation was performed by the staff surgeon who performed the procedure. Those patients who met clinical criteria for DCP were included. This was defined as a unilateral or bilateral worsening of motor function specific to a given myotome or set of myotomes detected on postoperative examination not directly attributable to intraoperative trauma such as misplaced instrumentation or inadvertent root injury. Motor strength examination information was gleaned from postoperative documentation by the primary neurosurgical team, including neurosurgery residents and staff surgeons. There is no standard postoperative CT/MRI, but all patients receive baseline radiography after fusion operations. Patients were excluded if they underwent revision of their instrumentation, had only sensory symptoms, or had any intraoperative complication relating to the nerve root causing immediate postoperative weakness.

\section{Data Collection}

Cost information was obtained using an institutionspecific program that calculates total hospital costs for a specific patient encounter. These are not patient charges; they are solely estimated costs for a list of services provided during hospitalization. These costs include surgical and anesthesia fees, postoperative recovery unit fees, perioperative antibiotics, per-diem rates for staying in general care or in an intensive care unit setting, physical therapy consultation, and other services rendered during a typical hospitalization. Cost information is generated as a list of costs per hospital day. Due to the wide range of cervical fusion operations performed in the study group, the operating room (OR) costs needed to be separated from the postoperative hospital-related costs, which is what we hypothesized to be higher in the DCP group. None of the patients were hospitalized prior to surgery, and therefore the total cost of the 1st day became the OR-related costs. All subsequent charges were listed as postoperative hospital-related costs. The operative- and nonoperative-related costs were compared with a matched control group who underwent surgery during the same timeframe. This control group was generated from the non-DCP group from 2008 to 2012 who underwent fusion operations. The groups were matched according to age, sex, and surgical approach. All costs and services were reflective of the standard costs for the current year. Due to institutional policy prohibiting publication of specific cost figures, data are presented using ratios of raw cost data. The raw cost data were tested using a paired t-test, and postoperative service utilization and complication data were tested using a Fisher's exact test with a p value $<0.05$ reported to be significant.

\section{Results}

We identified 1330 cases that were performed during the study period. Of those cases, 690 (51.9\%) were cervical decompressions with fusion. We identified 27 patients (18 men, 9 women) who underwent fusion and developed a DCP over the study period, for an incidence of 3.9\%. Indications for surgery included myelopathy in 11 patients, radiculopathy in 7 , myeloradiculopathy in 5 , central cord syndrome in 2, pseudarthrosis in 1, and torticollis in 1 . There were $19(70 \%)$ of 27 patients who underwent a posterior approach alone, 6 (22\%) of 27 who had an anterior approach alone, and 2 (7\%) of 27 who had combined anterior/posterior fusions. Ten different surgeons performed the procedures. Delayed radiculopathies were unilateral in $18(67 \%)$ of 27 patients, and prophylactic foraminotomies had been performed in $13(48 \%)$ of 27 patients. The most common myotome affected was the C-5 myotome in 20 patients $(74 \%)$, and 13 patients $(48 \%)$ had multiple myotomes affected. Discharge location was home in 22 patients $(81 \%)$, with 5 patients $(19 \%)$ requiring acute rehabilitation stays. At the final follow-up (mean 12.5 months, median 10 months), 11 patients (41\%) experienced complete resolution of the deficit, 7 (26\%) experienced significant improvement to that point, and $9(33 \%)$ had persistent weakness. Long-term follow-up data were available in every patient at the time of this analysis.

These 27 patients were compared with 24 controls (15 men, 9 women) who underwent fusion during the same time period (Table 1). Indications for surgery in the control group included myelopathy in 12 controls, radiculopathy in 5, myeloradiculopathy in 5, pseudarthrosis in 1 , and revision fusion in 1 . There was no significant difference between the study patients and controls in mean age $(62.4 \pm 3.1$ years vs $63.8 \pm 2.5$ years, respectively; $p=$ $0.74)$, $\operatorname{LOS}(4.2 \pm 3.3$ days vs $3.8 \pm 4.5$ days, respectively; $\mathrm{p}=0.43)$, presence of other complication $(4 / 27$ vs $2 / 24$,

TABLE 1. Case-matched cost analysis

\begin{tabular}{lccc}
\hline \multicolumn{1}{c}{ Variable } & $\begin{array}{c}\text { Delayed } \\
\text { Radiculopathy }\end{array}$ & Controls & p Value \\
\hline Mean age \pm SD (yrs) & $62.4 \pm 3.1$ & $63.8 \pm 2.5$ & 0.74 \\
\hline Mean LOS \pm SD (days) & $4.2 \pm 3.3$ & $3.8 \pm 4.5$ & 0.43 \\
\hline Mean OR-related costs & $1.08 \pm 0.09$ & $1.0 \pm 0.07$ & $0.58 \dagger$ \\
\hline Mean hospital-related costs* & $1.67 \pm 0.15$ & $1.0 \pm 0.09$ & $0.04 \ddagger$ \\
\hline
\end{tabular}

* Due to institutional policy prohibiting publication of specific cost figures, data are presented using ratios of raw cost data.

$\dagger$ Mean difference $\$ 2605.30$.

$\ddagger$ Mean difference $\$ 2895.27$. 
respectively; $\mathrm{p}=0.67)$, and OR-related costs $(1.08 \pm 0.09$ vs $1.0 \pm 0.07$, respectively; $p=0.58$ ). There was a significant difference in nonoperative hospital-related costs between the study patients and controls $(1.67 \pm 0.15$ vs $1.0 \pm$ 0.09 , respectively; $\mathrm{p}=0.04)$. Postoperative imaging $(\mathrm{CT} /$ MRI) was obtained significantly more often in DCP patients $(14 / 27,52 \%)$ compared with control patients $(4 / 24$, $17 \% ; \mathrm{p}=0.018)$. Physiatry consultation was also used significantly more often in DCP patients $(24 / 27,89 \%)$ than in controls $(15 / 24,63 \% ; \mathrm{p}=0.046)$.

\section{Discussion}

Delayed cervical palsies are a well-recognized yet poorly understood complication of cervical spine surgery. While the true origin remains elusive, it is likely a multitude of factors that ultimately lead to this complication. This complication occurs in nearly $5 \%$ of patients, and deficits may be permanent in as many as $19 \%$ of patients. ${ }^{8,9}$ Like any postoperative complication, DCPs result in higher hospital-related costs following surgery. In this study, there was no difference in OR-related costs or LOS between the study group and the control group, but there was a significant difference in postoperative hospital-related costs between these groups, with overall hospital costs increasing by approximately $\$ 3000$ for patients experiencing DCPs. We demonstrated that postoperative imaging, including CT/MRI studies, were used significantly more often in the DCP group compared with the control group. There was also a significantly higher utilization of physiatry services in the DCP group. There are likely other factors involved in the care of DCP patients that add to the increase in cost, but imaging and physiatry are higher-cost services, which likely drive the majority of the cost disparity. There was no difference in other postoperative complications between groups, which could have introduced a confounding bias into the findings. Hospital-related costs only convey a portion of the true costs of DCPs as increased costs likely continue after hospitalization in the form of more frequent follow-up, utilization of rehabilitation programs, and further diagnostic testing performed in these patients, along with the societal costs related to disability and time away from work. This increase in cost is an important factor to consider as many patients undergoing surgery for cervical spondylotic myelopathy are Medicare beneficiaries, and hospitals need to be aware of factors increasing the cost of providing care to these patients and how best to avoid them, as an increase in cost does not always translate into an increase in hospital reimbursement.

Certainly it is expected that there will be an increase in postoperative hospital-related costs when a patient experiences a complication such as a DCP, but the fact that this did not significantly increase the LOS, and also that the majority of these palsies resolved over time, is interesting. When faced with the presentation of an idiopathic DCP in a postsurgical patient, many surgeons would elect to perform multiple studies to delineate the nature of the complication, resulting in an increase in hospital cost. If it is known that there is no instrumentation malposition, failure, or other contributing operative complica- tion, further studies could potentially be avoided in these patients, decreasing the cost of the hospitalization. With the knowledge that long-term outcomes of DCP are favorable in many cases, the desire to monitor the patient in the hospital for more time could also be avoided, which would also decrease costs. This complication is just one of several known complications that are encountered in cervical spine surgery, and a better understanding of the specifics of each complication-specifically their modification of cost, hospital LOS, and return to work-are important factors for counseling patients and hospital care decision-making in an era when cost containment or cost awareness is becoming more important.

This study is limited due to the small number of study patients with the complication and the retrospective nature of the analysis. With a fairly small cost disparity in nonoperative hospital-related costs between groups relative to the overall cost of treatment, a larger case-control study could show either a lack of significant difference in costs or a larger disparity in costs presented in this study. A larger cohort could conversely show significant differences in hospital LOS. Additionally, costs and practices vary between institutions and regions. Thus, the results could potentially vary among institutions. Finally, cost analysis in this study ends with the patient leaving the hospital, making it impossible to further analyze the difference in cost to the system at time points extending beyond the end of the hospitalization. Many patients with this complication may miss work for extended periods of time and use medical services much more than patients who do not experience this complication. Unfortunately, the institutional program that generates cost data is not able to determine these costs, which are likely much higher than the reported increase in hospital-related costs from this study. Further studies will need to be performed to better determine potential origins of this complication, overall cost increases, and how best to avoid them in the future.

\section{Conclusions}

Delayed cervical palsy after cervical spine surgery does not increase hospital LOS, but does increase postoperative hospital-related costs in patients undergoing cervical spine fusion operations.

\section{References}

1. Bose B, Sestokas AK, Schwartz DM: Neurophysiological detection of iatrogenic C-5 nerve deficit during anterior cervical spinal surgery. J Neurosurg Spine 6:381-385, 2007

2. Chiba K, Toyama Y, Matsumoto M, Maruiwa H, Watanabe M, Hirabayashi K: Segmental motor paralysis after expansive open-door laminoplasty. Spine (Phila Pa 1976) 27:2108-2115, 2002

3. Currier BL: Neurological complications of cervical spine surgery: C5 palsy and intraoperative monitoring. Spine (Phila Pa 1976) 37:E328-E334, 2012

4. Hasegawa K, Homma T, Chiba Y: Upper extremity palsy following cervical decompression surgery results from a transient spinal cord lesion. Spine (Phila Pa 1976) 32:E197E202, 2007

5. Hojo Y, Ito M, Abumi K, Kotani Y, Sudo H, Takahata M, 
et al: A late neurological complication following posterior correction surgery of severe cervical kyphosis. Eur Spine J 20:890-898, 2011

6. Hosono N, Miwa T, Mukai Y, Takenaka S, Makino T, Fuji T: Potential risk of thermal damage to cervical nerve roots by a high-speed drill. J Bone Joint Surg Br 91:1541-1544, 2009

7. Imagama S, Matsuyama Y, Yukawa Y, Kawakami N, Kamiya M, Kanemura T, et al: C5 palsy after cervical laminoplasty: a multicentre study. J Bone Joint Surg Br 92:393-400, 2010

8. Nassr A, Eck JC, Ponnappan RK, Zanoun RR, Donaldson WF III, Kang JD: The incidence of C5 palsy after multilevel cervical decompression procedures: a review of 750 consecutive cases. Spine (Phila Pa 1976) 37:174-178, 2012

9. Sakaura H, Hosono N, Mukai Y, Ishii T, Yoshikawa H: C5 palsy after decompression surgery for cervical myelopathy: review of the literature. Spine (Phila Pa 1976) 28:24472451,2003

10. Sasai K, Saito T, Akagi S, Kato I, Ohnari H, Iida H: Preventing C5 palsy after laminoplasty. Spine (Phila Pa 1976) 28:1972-1977, 2003

11. Tanaka N, Nakanishi K, Fujiwara Y, Kamei N, Ochi M:
Postoperative segmental C5 palsy after cervical laminoplasty may occur without intraoperative nerve injury: a prospective study with transcranial electric motor-evoked potentials. Spine (Phila Pa 1976) 31:3013-3017, 2006

12. Yonenobu K, Hosono N, Iwasaki M, Asano M, Ono K: Neurologic complications of surgery for cervical compression myelopathy. Spine (Phila Pa 1976) 16:1277-1282, 1991

\section{Author Contributions}

Conception and design: Puffer, Mallory. Acquisition of data: Puffer, Clifton, Mallory. Analysis and interpretation of data: Puffer. Drafting the article: Clarke, Puffer, Mallory. Critically revising the article: Clarke, Puffer, Mallory. Reviewed submitted version of manuscript: all authors. Statistical analysis: Puffer.

\section{Correspondence}

Michelle J. Clarke, Department of Neurosurgery, Mayo Clinic, 200 1st St. SW, Rochester, MN 55905.email: clarke.michelle@ mayo.edu. 